\title{
Curcumin Loaded Nano Cubosomal Hydrogel: Preparation, In Vitro Characterization and Antibacterial Activity
}

\author{
A. ARCHANA, K. VIJAYA SRI*, M. MADHURI and CH. AJAY KUMAR \\ Malla Reddy College of Pharmacy, Maisammaguda, Secunderabad-500 014, \\ Andhra Pradesh, India
}

vijayasree_2002@yahoo.co.in

Received 20 July 2014 / Accepted 18 August 2014

\begin{abstract}
The objective of present investigation was to develop curcumin loaded cubosomes and evaluated for topical delivery. Curcumin-loaded cubosomes were prepared by homogenization method and prepared curcumin nano cubosomes were evaluated regarding microscopic studies, particle size analysis, zeta potential, entrapment efficiency (EE\%). Cubosomes hydrogels were prepared by using carbopol as gelling agent and evaluated for appearance, $\mathrm{pH}$, viscosity, entrapment efficiency, in vitro drug diffuse studies, skin irritation studies and anti bacterial activity. The particle size of the formulation was about $75.2 \mathrm{~nm}$ and zeta potential $-24 \mathrm{mv}$. The developed curcumin loaded cubosomal hydrogel gave rise to stable, nano-sized vesicles, able to improve curcumin anti bacterial activity in topical drug delivery.
\end{abstract}

Keywords: Curcumin, Glycerol mono oleate, Cubosome, Hydrogel, Characterization, Antibacterial activity

\section{Introduction}

Cubosomes are self-assembled liquid crystalline particles with a microstructure that provide unique properties in a size range of $50-10000 \mathrm{~nm}^{1}$. They are formed by dispersion of bicontinuous cubic liquid crystalline phases ${ }^{2}$. Bicontinuous cubic liquid crystalline phase is an optically clear, very viscous material that has a unique structure at the nanometer scale. The word 'bicontinuous' refers to the division of the two continuous but non-intersecting aqueous regions by a lipid bilayer that is contorted into a space-filling structure. Hydrating a surfactant or polar lipid that forms cubic phase and then dispersing the solid-like phase into smaller particles usually forms cubosomes. Cubosomes are the liquid crystalline cubic nano particles share features from both liquids and crystalline substances. Due to their intermediate state they are also called as "mesophases". Liquid crystalline nano particles possess nano cavities (aqueous medium which is separated by lipid bilayer's). In this view, system can be used as a carrier for hydrophilic as well as for lipo-philic drug molecules, peptides and proteins ${ }^{3}$.

Curcumin, a polyphenolic compound is an active principle of the perennial herb Curcuma longa (commonly known as turmeric). Curcumin has been shown to exhibit diverse pharmacologic effects antioxidant, anti-inflammatory, antimicrobial, anticarcinogenic, 
hepato- and nephro-protective, thrombosis suppressing, myocardial infarction protective, hypoglycemic and antirheumatic ${ }^{4,5}$. The reasons for reduced bioavailability of curcumin within the body include low intrinsic activity, spoor absorption, limited tissue distribution, short half-life, high rate of metabolism, inactivity of metabolic products and/or rapid elimination and clearance from the body ${ }^{6}$. The main aim of the present work is the preparation of curcumin-loaded cubosomes, evaluation and then incorporation of the curcumin-loaded cubosomes. The main objective of the present work is to enhance the bioavailability of curcumin by enhancing its absorption by formulating as cubosomes.

\section{Experimental}

Curcumin was purchased from S.D. Fine chemical Pvt Ltd. Mumbai. Propylene glycol, glycerine, potassium hydroxide were obtained from Accord labs, Secunderabad. The polymers polaxamer 407, glyceryl monooleate and carbopol were obtained from Dr.Reddy's laboratories, Hyderabad. All reagents used were of analytical grade.

\section{Preparation of cubosomes}

1.5 g Glycerol monooleate $(4.5 \% \mathrm{w} / \mathrm{w})$ and $0.25 \mathrm{~g}$ poloxamer $407(0.5 \% \mathrm{w} / \mathrm{w})$ were molten in a water bath. The drug (50 mg) was added to this molten mixture. The mixture was added drop wise into $50 \mathrm{~mL} \mathrm{pH} 7.4$ phosphate buffer (95\% w/w) at $70{ }^{\circ} \mathrm{C}$ under mechanical stirring at $1500 \mathrm{rpm}$. Dispersions were maintained under stirring at room temperature for $2 \mathrm{~h}$. Afterwards the dispersions were subjected to homogenization at $15000 \mathrm{rpm}$ at $60{ }^{\circ} \mathrm{C}$ for $1 \mathrm{~min}$. After cooling, the dispersions were maintained at room temperature in glass vials ${ }^{7}$.

\section{Characterization of cubosomes}

\section{Optical microscopy}

The prepared cubosomal formulation was spread on a glass slide and observed under optical microscope. The structure of cubosomes was examined with varied magnification powers (10x, 30x and 40x). Photomicrographs were taken using digital camera.

\section{Entrapment efficiency}

Entrapment efficiency was determined by centrifugation method. $5 \mathrm{~mL}$ of cubosomal formulation was taken and centrifuged to separate the unentrapped drug from cubosomes ${ }^{7}$. The supernatant liquid was taken and the absorbance was determined by using UV-spectrophotometer at $428 \mathrm{~nm}$. The entrapment efficiency was expressed as the percentage of drug entrapped into cubosomes which was determined by using the formula: Entrapment efficiency $=$ drug entrapped/total drug*100

\section{Particle size and Zeta potential analysis}

The particle size and zeta potential were determined by taking suitably diluted cubosomal formulation using Nano particle analyzer SZ-10 and SZ-100 respectively. The temperature was set at $25{ }^{\circ} \mathrm{C}$. Electrophoretic mobility and mean zeta potential values were obtained directly from the measurement.

\section{Preparation of hydrogels}

Two hydrogel formulations (HC1, HC2) were prepared with curcumin cubosomal formulation and another two (HD1, HD2) were prepared with pure curcumin. The four hydrogels (HC1, HC2, HD1, HD2) were prepared with $0.5 \%$ and $1 \%$ of carbopol alternatively. The carbopol was dissolved in $10 \mathrm{ml}$ of water and stirred well to form gel. $5 \mathrm{~mL}$ 
of propylene glycol and $2 \mathrm{~mL}$ of glycerine were added to the formulation. $10 \mathrm{~mL}$ of cubosomal formulations (HC1, HC2) or drug solution (HD1, HD2) were incorporated into gel and carefully stirred manually.

\section{Characterization of cubosomal hydrogels}

The prepared curcumin loaded hydrogels were evaluated for $\mathrm{pH}$, skin irritation and in-vitro skin permeation and skin deposition.

Appearance, $p H$, viscosity

$\mathrm{pH}$ of the prepared nano cubosomal hydrogels was determined by using $\mathrm{pH}$ meter. Take sufficient quantity of hydrogel and diluted with water to form aqueous dispersion. Viscosity of prepared gels was measured by Brookfield-Viscometer.

\section{Skin irritation studies}

This study was carried out on healthy Wistar rats. The animals were divided into 3 groupscontrol, hydrogels with cubosomes, hydrogels with pure drug. The back skin was shaved before one day of starting the study. Formulations were applied to assess the skin sensitizing pot. At the end of study, the animals were observed and scores were given for any skin irritation like erythema or edema and score given as per the irritation.

\section{In vitro skin permeation studies}

In vitro skin permeation studies were performed by using goat ear skin. The superficial skin was collected from the back of goat ear and the hair on the skin was removed. The dermal side of the skin was thoroughly cleaned of any adhering tissues ${ }^{8}$. Dermis part of the skin was wiped 3 to 4 times with a wet cotton swab soaked in isopropanol to remove any adhering fat material. The skin specimens were cut into appropriate size after carefully removing subcutaneous fat and washing with normal saline. Skin was mounted in a modified Franz diffusion cell, kept at $37^{\circ} \mathrm{C}$. Weighed quantity of cubosomal gel was spreaded on the stratum corneum side of skin (donor compartment) and dermis side was facing receptor compartment. Receptor compartment contains $100 \mathrm{~mL}$ of $\mathrm{pH} 7.4$ phosphate buffer and for every one hour $1 \mathrm{~mL}$ of sample was taken and replaced with the same volume of fluid. After $24 \mathrm{~h}$ sampling, absorbance was measured at $428 \mathrm{~nm}$ against blank of $\mathrm{pH} 7.4$ phosphate buffer by UV spectrophotometer. The flux was calculated using following formula,

$$
\text { Flux }(J)=\frac{\text { slop }}{\text { thickness of skin / partition coefficient of drug }}
$$

Partition coefficient of drug $=3.2$

$$
\text { Permeation coefficient, } K p=\frac{\text { flux }}{\text { amount of drug in the formulation }}
$$

\section{In-vitro skin deposition studies}

At the end of the permeation experiments (after $24 \mathrm{~h}$ ), the skin was removed. The remaining formulation adhering to the skin was scraped with a spatula. The skin surface was washed with ethanol: PBS (1:1) and then with water to remove excess drug from surface ${ }^{8}$. The skin was then cut into small pieces. The tissue was further washed with ethanol: PBS (1:1) and left for $6 \mathrm{~h}$ at room temperature. Then the washed tissue was subjected to centrifugation for $10 \mathrm{~min}$. Then the curcumin content was analyzed by UV visible spectrophotometric method at $428 \mathrm{~nm}$. 


\section{Kinetic analysis of diffusion data}

In order to describe the kinetics of the release process of drug in the different formulations, zero- order $(\mathrm{Qt}=\mathrm{Q} 0+\mathrm{K} 0 \mathrm{t})$, first order $(\ln \mathrm{Qt}=\ln \mathrm{Q} 0+\mathrm{K} 1 \mathrm{t})$, Higuchi $\left(\mathrm{Qt}=\mathrm{KH} \mathrm{t} \mathrm{t}_{1 / 2}\right)$ and Korsmeyer- Peppas (Qt/Q8= Ktn) models were fitted to the diffusion data of hydrogel formulations using linear regression analysis ${ }^{9}$. A value of $n=0.5$ indicates case I (Fickian) diffusion or square root of time kinetics, $0.5<\mathrm{n}<1$ anomalous (non- Fickian) diffusion, $\mathrm{n}=1$ Case -II transport and $\mathrm{n}>1$ Super Case II transport.

\section{Antibacterial activity of cubosomal gel containing curcumin}

A sterile borer was used to prepare cups of $10 \mathrm{~mm}$ diameter in the agar media spread with the microorganisms. $0.1 \mathrm{~mL}$ of inoculums (of 104 to $106 \mathrm{CFU} / \mathrm{mL}$ population prepared from standardized culture, adjusted with peptone water) was spread on the agar plate by spread plate technique. Measured amount of each sample and standard samples were added to the cups. All the plates were kept in a refrigerator at 2 to $8{ }^{\circ} \mathrm{C}$ for a period of two hours for effective diffusion of test compounds and standards. Later, they were incubated at $37^{\circ} \mathrm{C}$ for $24 \mathrm{~h}$. The presence of definite zones of inhibition around the cup indicated antibacterial activity. The diameter of the zone of inhibition was measured and recorded.

\section{Results and Discussion}

\section{Optical microscopy}

Morphology of cubosomes was studied under optical microscope. Cubosomes were found to be cubic in shape and crystalline in appearance as shown in Figure 1a.

\section{Particle size and zeta potential analysis}

Particle size and zeta potential of the formulation were determined using particle size analyzer. The particle size and zeta potential (Figure 1b) of formulation was found to be $75.2 \mathrm{~nm}$ and -24 $\mathrm{mV}$ respectively. The value of zeta potential indicates the presence of sufficient charge to inhibit aggregation of cubosomes due to electric repulsion. This indicates that the formulation is stable.

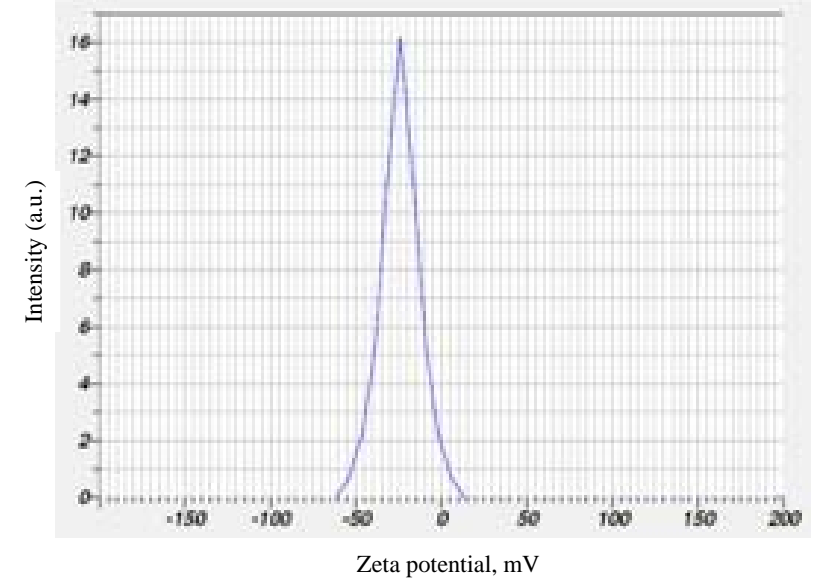

Figure 1a. Zeta potential of optimized formulation

\section{Entrapment efficiency}

The entrapment efficiency of cubosomal formulation was determined and the value is $86.4 \%$.
Figure 1b. Digital photomicro-

graphs of cubosomes

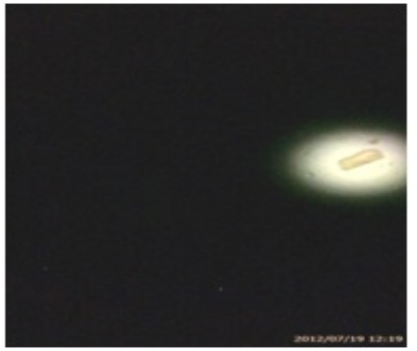


Appearance, Viscosity, $\mathrm{pH}$

The prepared cubosomal hydrogels were examined visually for their colour consistency and found to appear white, translucent gels without any lumps and aggregates.viscosity and $\mathrm{pH}$ of the hydrogels was found to be $10700 \mathrm{cPs}$ to $29100 \mathrm{cPs}$ and 7.3 to 7.1. This indicated that all formulations were compatible with the skin.

\section{Skin irritation studies}

When the selected formulations were applied to rats, no signs of erythema or redness were observed until 7 days.

\section{In-vitro skin permeation studies}

Skin permeation studies were carried out for all the four prepared hydrogels containing cubosomes and drug for $24 \mathrm{~h}$ using goat ear skin and the results are shown in Figure 2. The skin permeation of HC2 was found to be higher when compared to the remaining gels. It was also found that the hydrogels with cubosomes showed better permeability when compared to the hydrogels with pure drug. This shows that cubosomes enhance the peremeability of the drug.

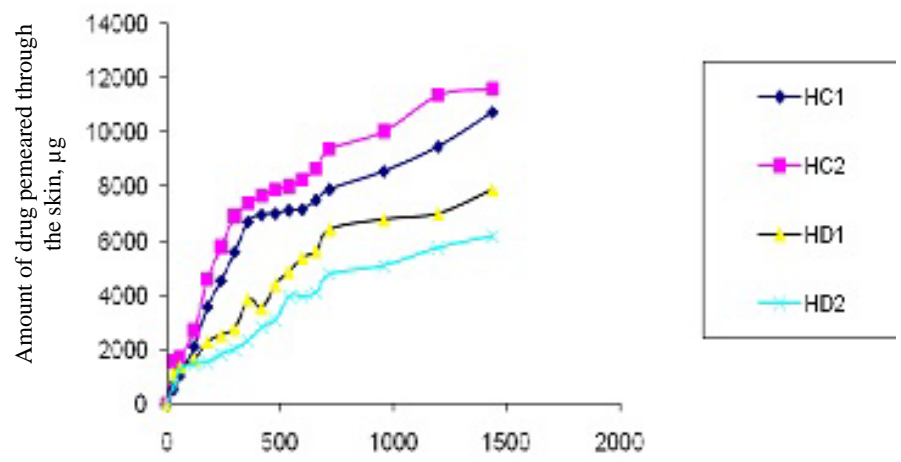

Figure 2. Comparative studies of different hydrogel

\section{In-vitro skin deposition studies}

After 24 h of skin permeation studies, skin deposition studies were performed and the amount of drug within the skin was calculated. The amount of the drug in the skin for the formulations HC1, HC2, HD1 and HD2 was found to be $7420 \pm 0.08,7318 \pm 0.07,1956 \pm 0.02$ and $1602 \pm 0.04 \mu$ g respectively. HC1 gel showed higher drug deposition in the skin when compared to other gels. For topical antimicrobials enhanced drug delivery and retention in the stratum corneum (the outer layer of skin) is desired.

\section{Kinetic analysis of diffusion data}

The drug release data were analysed for the type of release mechanism. Curve fitting analysis was done for all formulations to determine the type of drug release mechanism. For this, release kinetic studies of all formulations were studied for different kinetic equations (zero order, first order, Higuchi's model, korsmeyer-peppas model). The $r^{2}$ values for all formulations were shown in Table 1 . For the cubosomes and incorporated hydrogels, the best fit with the higher correlation was found with the Higuchi's model for all formulations. For the cubosomes incorporated hydrogel, the $r^{2}$ was found to be 0.987 with the ' $n$ ' value 
was 2.091. Here ' $n$ ' values were more than 1 , so it concluded that the drug release mechanism was followed super case-II transport mechanism, which concluded that the drug release was affected by erosion of polymer.

Table 1. Kinetic analysis of diffusion data

\begin{tabular}{ccccc}
\hline \multirow{2}{*}{$\begin{array}{c}\text { Formulation } \\
\text { code }\end{array}$} & Zero order & First order & Higuchi model & Peppas model \\
\cline { 2 - 5 } HC1 & 0.8347 & 0.7260 & 0.9442 & 0.9086 \\
HC2 & 0.8270 & 0.5140 & 0.9625 & 0.9168 \\
HD1 & 0.7996 & 0.8541 & 0.9614 & 0.9026 \\
HD2 & 0.8376 & 0.8104 & 0.9026 & 0.6680 \\
\hline
\end{tabular}

Anti bacterial activity

The antimicrobial efficacy of cubosomes was significantly higher when compared to pure curcumin. Zone of inhibition (mm) for cubosomes was found to be $16.20 \pm 4.26 \mathrm{~mm}$ and $11.36 \pm 1.14 \mathrm{~mm}$ for pure curcumin at $24 \mathrm{~h}$. The zone of inhibition for cubosome incorporated hydrogel was higher compared to the pure curcumin, which is shown in Figure 3.

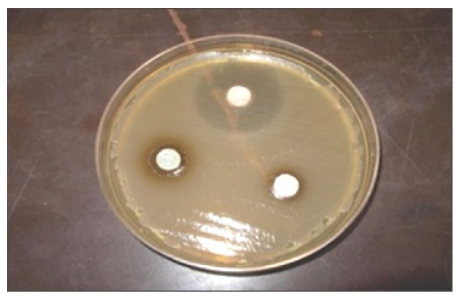

Figure 3. Culture plate showing Zones Inhibition for Escherichia Coli

\section{Conclusion}

The developed curcumin loaded cubosomal hydrogel gave rise to stable, nano-sized vesicles, able to improve curcumin anti bacterial activity in topical drug delivery.

\section{References}

1. Garg G, Saraf S and Saraf S, Biol Pharm Bull., 2007, 30(2), 350-353.

2. $\quad$ Chung H, Kim J, Um J Y, Kwon I C and Jeong S Y, Diabetologia., 2002, 45(3), 448-451.

3. Lynch M L, Ofori-Boateng A, Hippe A, Kochvar K and Spicer P T, J Colloid Interface Sci., 2003, 260(2), 404-413; DOI:10.1016/S0021-9797(02)00016-4

4. Strasser E M, Wessner B, Manhart N and Roth E, Biochem Pharmacol., 2005, 70(4), 552-559; DOI:10.1016/j.bcp.2005.05.030

5. Yang Z, Tan Y, Chen M, Dian L, Shan Z, Peng X and Wu C, AAPS Pharm Sci Tech., 2012, 13(4), 1483-1491; DOI:10.1208/s12249-012-9876-2

6. Kurien B T and Scofield R H, Trends Pharmacol Sci., 2009, 30(7), 334-335; DOI:10.1016/j.tips.2009.04.005

7. Vijaya Sri K, Arachana A, Ajay Kumar Ch and Vinay jain G, Indo American J Pharm Res., 2014, 4(2), 992-999.

8. Vinod K R, Sravya K, Sandhya S, David Banji, Anbazhagan S and Prameela Rani A, J Chine Pharm Sci., 2013, 22(4), 303-313.

9. Korsmeyer R W, Gurny R, Doelker E, Buri P and Peppas N A, Int J Pharm., 1983, 15(1), 25-35; DOI:10.1016/0378-5173(83)90064-9 